\title{
The semi-index product formula
}

\author{
by
}

Jerzy Jezierski (Warszawa)

Abstract. We consider fibre bundle maps

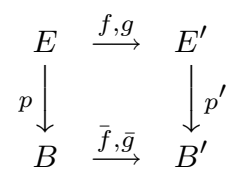

where all spaces involved are smooth closed manifolds (with no orientability assumption). We find a necessary and sufficient condition for the formula

$$
|\operatorname{ind}|(f, g: A)=|\operatorname{ind}|(\bar{f}, \bar{g}: p(A))|\operatorname{ind}|\left(f_{b}, g_{b}: p^{-1}(b) \cap A\right)
$$

to hold, where $A$ stands for a Nielsen class of $(f, g), b \in p(A)$ and |ind| denotes the coincidence semi-index from [DJ]. This formula enables us to derive a relation between the Nielsen numbers $N(f, g), N(\bar{f}, \bar{g})$ and $N\left(f_{b}, g_{b}\right)$.

Introduction. In [DJ] the Nielsen theory was extended to coincidences of pairs of maps $f, g: M \rightarrow N$ for $M, N$ closed manifolds of the same dimension (with no orientability assumption). In this paper we discuss the "Nielsen number product formula" as in $[\mathrm{Y}]$ and [Je].

After recalling in Section 1 the main results of [Y], [Je] and [DJ] we consider in Section 2 "self-reducing coincidence points". Nielsen classes containing such points appear only in the non-orientable case. They turn out to be the obstruction to the semi-index product formula which is discussed in Section 3. In Section 4 we prove a Nielsen number product formula (Thm. (4.3)), and in the last section we get a formula for the coincidence Nielsen number of pairs of maps between some $K(\pi, 1)$ spaces (Corollary (5.5), Remark (5.6)).

1. Preliminaries. We begin by recalling the definitions from $[\mathrm{Y}]$ and [Je]. Let $u$ and $v$ be paths in a topological space $Y$ such that $u(1)=v(0)$. Then $u+v$ denotes their composition and $-u$ the path opposite to $u$. Let $H$ 
be a normal subgroup of the fundamental groupoid $\pi_{1} Y$, i.e. for any $y \in Y$ there is a normal subgroup $H(y)$ of $\pi_{1}(Y, y)$ such that for any path $r$ joining $y$ and $y^{\prime}$ the isomorphism $\pi_{1}(Y, y) \ni\langle a\rangle \rightarrow\langle-r+a+r\rangle \in \pi_{1}\left(Y, y^{\prime}\right)$ carries $H(y)$ onto $H\left(y^{\prime}\right)$. The paths $u$ and $v$ are called H-homotopic iff $u(0)=v(0)$, $u(1)=v(1)$ and $\langle u-v\rangle \in H(u(0))$. We then write $u \stackrel{H}{\simeq} v$. The $H$-homotopy class of the path $u$ is denoted by $\langle u\rangle_{H}$.

Let $X$ be another topological space and let $f, g: X \rightarrow Y$ be a pair of continuous maps. Let $\Phi(f, g)=\{x \in X: f x=g x\}$ denote the coincidence set of these maps. The points $x, y \in \Phi(f, g)$ will be called H-Nielsen equivalent if there exists a path $u$ joining them such that $f u \stackrel{H}{\simeq} g u$. We denote the quotient set by $\Phi_{H}^{\prime}(f, g)$ and call its elements $H$-Nielsen classes. We omit $H$ if $H=0$.

Fix $x \in X$ and a path $r$ joining $f x$ and $g x$ in $Y((x, r)$ will be called a reference pair $)$. We define an action of $\pi_{1}(X, x)$ on $\pi_{1}(Y, f x) / H(f x)$ by

$$
\left(\langle d\rangle,\langle a\rangle_{H}\right) \rightarrow\langle f d+a+r-g d-r\rangle_{H}
$$

where $\langle d\rangle \in \pi_{1}(X, x),\langle a\rangle_{H} \in \pi_{1}(Y, f x) / H(f x)$. We denote by $\nabla_{H}(f, g$ : $x, r)$ the set of all orbits of the above action and by $\left[\langle a\rangle_{H}\right]$ the orbit of $a$.

The following lemma establishes a connection between the sets $\Phi_{H}^{\prime}(f, g)$ and $\nabla_{H}(f, g: x, r)$.

(1.1) Lemma $((1.2)$ in [Je], see also $[\mathrm{Y}])$. For any $x_{0} \in \Phi(f, g)$ the set $\left\{\langle f u-g u-r\rangle_{H}: u\right.$ is a path from $x$ to $\left.x_{0}\right\}$ is an orbit of the above action. Moreover, two coincidence points determine the same orbit iff they are $H$ Nielsen equivalent.

The above lemma determines an injection

$$
\varrho(x, r): \Phi_{H}^{\prime}(f, g) \rightarrow \nabla_{H}(f, g: x, r) .
$$

Lemma (1.3) in [Je] allows us to identify the sets $\nabla_{H}(f, g: x, r)$ for all $(x, r)$ so that the $\varrho(x, r)$ induce a canonical injection

$$
\varrho: \Phi_{H}^{\prime}(f, g) \rightarrow \nabla_{H}(f, g) .
$$

Now we recall the notion of coincidence semi-index (see [DJ] for details). Let $M, M^{\prime}$ be smooth closed $m$-manifolds, $f, g: M \rightarrow M^{\prime}$ smooth transverse maps and let $x_{0}, x_{1} \in \Phi(f, g)$. We will say that $x_{0}$ and $x_{1}$ are $R$-related iff there is a path $u$ from $x_{0}$ to $x_{1}$ such that $f u \simeq g u$ and exactly one of the paths $u$ or $f u$ is orientation-preserving. We then say that $u$ is graphorientation-reversing (cf. Definition (1.2) in [DJ]) and we write $x_{0} R x_{1}$ (in [DJ] $x_{0}$ was said to reduce $\left.x_{1}\right)$.

Let $A \subset \Phi(f, g)$. We call $A=\left\{a_{1}, b_{1}, \ldots, a_{k}, b_{k}: c_{1}, \ldots, c_{s}\right\}$ a decomposition iff (i) $a_{i} R b_{i}(i=1, \ldots, k)$, (ii) no $\left\{c_{i}, c_{j}\right\}$ are $R$-related $(i, j=$ $1, \ldots, s, i \neq j)$. 
If only (i) holds we call $A$ an incomplete decomposition. Sometimes we also write $A=A_{0} \cup\left\{c_{1}, \ldots, c_{s}\right\}$ and we call the elements $\left\{c_{1}, \ldots, c_{s}\right\}$ free in this decomposition. We define the semi-index of the set $A$ as the number of free points in its decomposition and denote it by $\mid$ ind $\mid(f, g: A)$. Finally, we call a Nielsen class $A \in \Phi_{H}^{\prime}(f, g)$ essential iff $\mid$ ind $\mid(f, g: A) \neq 0$ and define the $H$-Nielsen number $N_{H}(f, g)$ as the number of essential classes.

In Section 3 we will need the following version of (1.3) of [DJ].

(1.2) Lemma. Let $A \subset \Phi(f, g)$ and let $\mathfrak{A}: A=A_{0} \cup\left\{c_{1}, \ldots, c_{s}\right\}, \mathfrak{A}^{\prime}$ : $A=A_{0}^{\prime} \cup\left\{c_{1}^{\prime}, \ldots, c_{s}^{\prime}\right\}$ be two decompositions. Then there exists a bijection $\phi:\left\{c_{1}, \ldots, c_{s}\right\} \rightarrow\left\{c_{1}^{\prime}, \ldots, c_{s}^{\prime}\right\}$ such that for any $c_{i}$ there exists a graphorientation-preserving path $u$ from $c_{i}$ to $\phi\left(c_{i}\right)$.

Proof. Set $a_{1}=c_{i}$. The proof of (1.3) in [DJ] gives us a sequence $a_{1}, \ldots, a_{2 k+1}$ where $a_{2 i-1}, a_{2 i}$ form a pair in $\mathfrak{A}^{\prime}, a_{2 i}, a_{2 i+1}$ form a pair in $\mathfrak{A}$ $(i=1, \ldots, k)$ and $a_{2 k+1}$ is free in $\mathfrak{A}^{\prime}$. We put $\phi\left(c_{i}\right)=a_{2 k+1}$. We notice that $a_{i} R a_{i+1}$, hence there is a graph-orientation-reversing path $u_{i}$ joining them. Now the composition $u=u_{1}+\ldots+u_{2 k}$ is graph-orientation-preserving.

(1.3) Lemma. Consider the diagram

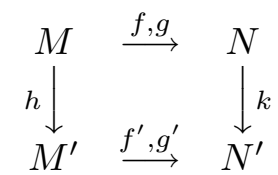

of path-connected spaces. Let $H \subset \pi_{1} N, H^{\prime} \subset \pi_{1} N^{\prime}$ be normal subgroups such that $k_{\#} H \subset H^{\prime}$.

(a) If the diagram is commutative then it determines a map $\varkappa: \nabla_{H}(f, g)$ $\rightarrow \nabla_{H^{\prime}}\left(f^{\prime}, g^{\prime}\right)$ given by

$$
\varkappa: \nabla_{H}(f, g: x, r) \rightarrow \nabla_{H^{\prime}}\left(f^{\prime}, g^{\prime}: h x, k r\right), \quad \varkappa\left[\langle a\rangle_{H}\right]=\left[\langle k a\rangle_{H^{\prime}}\right] .
$$

(b) If the diagram is homotopy commutative (by means of homotopies $\left.(F, G):(k f, k g) \simeq\left(f^{\prime} h, g^{\prime} h\right)\right), k$ and $h$ are homeomorphisms and $k_{\#} H=H^{\prime}$ then we have a bijective transformation $\eta: \nabla_{H}(f, g) \rightarrow \nabla_{H^{\prime}}\left(f^{\prime}, g^{\prime}\right)$ given by

$$
\begin{aligned}
\nabla_{H}(f, g: x, r) \ni\left[\langle a\rangle_{H}\right] \rightarrow & {\left[\langle-F(x, \cdot)+k a+F(x, \cdot)\rangle_{H^{\prime}}\right] } \\
& \in \nabla_{H^{\prime}}\left(f^{\prime}, g^{\prime}: h x,-F(x, \cdot)+k r+G(x, \cdot)\right) .
\end{aligned}
$$

(c) If the assumptions of (a) and (b) hold and the considered homotopies are constant then $\varkappa=\eta$.

(d) If the assumptions of (b) hold, and all spaces are closed smooth manifolds of the same dimension then $\eta$ is semi-index-preserving, i.e.

$$
\mid \text { ind }|(f, g: A)=| \operatorname{ind} \mid\left(f^{\prime}, g^{\prime}: \eta A\right) \quad \text { for any } A \in \nabla_{H}(f, g) \text {. }
$$


Proof. (a)-(c) were proved as Lemma (2.1) in [Je]. It was also shown there that our diagram may be considered as the composition of two squares

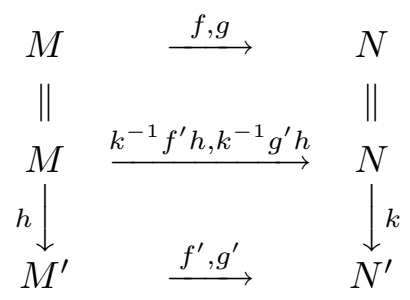

The upper square is homotopy commutative (with the homotopies $\left(k^{-1} F\right.$, $\left.k^{-1} G\right)$ ) and the lower one is commutative (with constant homotopies). Thus it is enough to show that the transformations $\varkappa_{1}, \varkappa_{2}$ induced by these diagrams are semi-index-preserving. But $\varkappa_{1}=\mu_{\left(k^{-1} F, k^{-1} G\right)}$ (for definition, see [Je, (1.6)]) and we apply (1.4) of [DJ]. On the other hand, it is evident that $\varkappa_{2}$ is semi-index-preserving since $h$ and $k$ are homeomorphisms.

Let now $(E, p, B),\left(E^{\prime}, p^{\prime}, B^{\prime}\right)$ be locally trivial bundles where the total spaces, base spaces and fibres are smooth connected closed manifolds of respectively equal dimensions. Denote the fibres by $E_{b}=p^{-1} b, E_{b^{\prime}}^{\prime}=p^{\prime-1} b^{\prime}$ and let $\lambda, \lambda^{\prime}$ denote the lifting functions of the Hurewicz fibrations $(E, p, B)$, $\left(E^{\prime}, p^{\prime}, B^{\prime}\right)$. Since the bundles are locally trivial, we may assume that for any path $\bar{u}$ in $B$ the map $\tau_{\bar{u}}: E_{\bar{u}(0)} \rightarrow E_{\bar{u}(1)}$ given by $\tau_{\bar{u}}(x)=\lambda(x, \bar{u})(1)$ is a homeomorphism.

Consider a commutative diagram

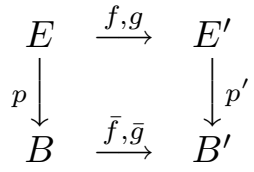

Denote by $f_{b}: E_{b} \rightarrow E_{\bar{f} b}^{\prime}, g_{b}: E_{b} \rightarrow E_{\bar{g} b}^{\prime}$ the restrictions of $f, g$. Let $b_{0}, b_{1} \in \Phi(\bar{f}, \bar{g})$ be Nielsen equivalent and let $\bar{u}$ be a path joining them and establishing the Nielsen relation. Then (Sect. 4 in [Je]) the diagram

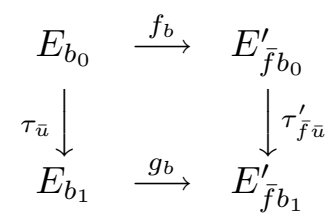

is homotopy commutative and the computations of Section 4 in [Je] give us a bijective transformation $T_{\bar{u}}: \nabla_{K}\left(f_{b_{0}}, g_{b_{0}}\right) \rightarrow \nabla_{K}\left(f_{b_{1}}, g_{b_{1}}\right)$ where $K=$ $\operatorname{ker}\left(\pi_{1} E_{b}^{\prime} \rightarrow \pi_{1} E^{\prime}\right)$. Moreover, Lemma (1.2) implies that $T_{\bar{u}}$ is semi-indexpreserving. This implies $N_{K}\left(f_{b_{0}}, g_{b_{0}}\right)=N_{K}\left(f_{b_{1}}, g_{b_{1}}\right)$ for $b_{0}, b_{1} \in \Phi(\bar{f}, \bar{g})$ in 
the same Nielsen class.

2. Self-reducing coincidence points. Let $f, g: M \rightarrow N$ be transverse. We will discuss the following problem: when does a coincidence point $x \in \Phi(f, g)$ satisfy $x R x$, i.e. when does there exist a graph-orientationreversing loop $a$ based at $x$. We will see that such points may only appear in the non-orientable case and that they are the only obstructions to the semi-index product formula discussed in the next section.

Let $M, N$ denote again smooth closed connected $n$-manifolds.

(2.1) Definition. Let $x \in \Phi(f, g)$ and let $H \subset \pi_{1} M, H^{\prime} \subset \pi_{1} N$ denote the subgroups of orientation-preserving elements. We define

$$
\begin{aligned}
C\left(f_{\#}, g_{\#}\right)_{x} & =\left\{a \in \pi_{1}(M, x): f_{\#} a=g_{\#} a\right\}, \\
C^{+}\left(f_{\#}, g_{\#}\right)_{x} & =C\left(f_{\#}, g_{\#}\right)_{x} \cap H .
\end{aligned}
$$

(2.2) Lemma. Let $f, g: M \rightarrow N$ be transverse and let $x \in \Phi(f, g)$. Then $x R x$ if and only if $C^{+}\left(f_{\#}, g_{\#}\right)_{x} \neq C\left(f_{\#}, g_{\#}\right)_{x} \cap f_{\#}^{-1}\left(H^{\prime}\right)$ (in other words, if there exists a loop a based at $x$ such that $f a \simeq g a$ and exactly one of the loops a or $f a$ is orientation-preserving).

P r o of. It suffices to recall the definition of $x R x$ and observe that

$$
\begin{array}{lll}
a \in C^{+}\left(f_{\#}, g_{\#}\right)_{x} & \text { means } & a \text { is orientation-preserving, } \\
a \in f_{\#}^{-1}\left(H^{\prime}\right) & \text { means } & f_{\#} a \text { is orientation-preserving. }
\end{array}
$$

A coincidence point $x$ satisfying $x R x$ will be called self-reducing.

(2.3) LEMMA. If a Nielsen class A contains a self-reducing point then any two points in this class are $R$-related, and thus

$$
\text { |ind } \mid(f, g: A)= \begin{cases}0 & \text { if } \# A \text { is even, } \\ 1 & \text { if } \# A \text { is odd }\end{cases}
$$

Proof. Let $x_{0} \in A$ be self-reducing and let $a$ be a graph-orientationreversing loop based at $x_{0}$. First we show that $x_{0} R x_{1}$ for any other $x_{1} \in A$. Since the two points lie in one Nielsen class, there exists a path $u$ joining them such that $f u \simeq g u$. Now, either $u$ or $a+u$ is graph-orientationpreserving, which implies $x_{0} R x_{1}$.

Let $x_{2} \in A$. Then $x_{1} R x_{0}, x_{0} R x_{0}, x_{0} R x_{2}$ and the odd transitivity implies $x_{1} R x_{2}$ (see [DJ, (1.3)]).

(2.4) ExAMPLE. Let $M$ be a non-orientable two-dimensional connected manifold. It may be regarded as a CW-complex with a unique 2-cell. Let $f^{\prime}: M \rightarrow S^{2}$ be a map which sends the 1-skeleton into a point $y_{1} \in S^{2}$ and the interior of the 2-cell diffeomorphically onto $S^{2}-y_{1}$. Let $g^{\prime}: M \rightarrow S^{2}$ denote the constant map with $g^{\prime}(M)=y_{0} \neq y_{1}$. Then the pair $\left(f^{\prime}, g^{\prime}\right)$ 
is transverse and $\Phi\left(f^{\prime}, g^{\prime}\right)$ consists of a single point $x_{0}$. This point is selfreducing since there exists an orientation-reversing loop at $x_{0}$ whose images by $f^{\prime}$ and $g^{\prime}$ are null homotopic.

Now we consider the maps $\bar{f}, \bar{g}: S^{2} \rightarrow S^{2}$ where $\bar{f}=\operatorname{id}_{S^{2}}, \bar{g}(x, y, z)=$ $(-x,-y, z)$. This pair is transverse and $\Phi(\bar{f}, \bar{g})=\{(0,0,1),(0,0,-1)\}=$ $\left\{b_{0}, b_{1}\right\}$ are two Nielsen equivalent points which are not $R$-related (since $S^{2}$ is orientable). Notice that $|\operatorname{ind}|\left(f^{\prime}, g^{\prime}\right)=1, N\left(f^{\prime}, g^{\prime}\right)=1$ and $\mid$ ind $\mid(\bar{f}, \bar{g})=2$, $N(\bar{f}, \bar{g})=1$. Consider the diagram

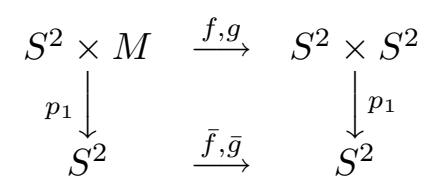

where $f=\bar{f} \times f^{\prime}, g=\bar{g} \times g^{\prime}$ and $p_{1}$ denotes the projection on the first factor. Then $\Phi\left(\bar{f} \times f^{\prime}, \bar{g} \times g^{\prime}\right)=\left\{\left(b_{0}, x_{0}\right),\left(b_{1}, x_{0}\right)\right\}$ and the two points are Nielsen equivalent. Each of them is self-reducing (since so is $x_{0} \in \Phi\left(f^{\prime}, g^{\prime}\right)$ ) and now Lemma (2.3) implies $\mid$ ind $\mid(f, g)=0$.

The example shows that the product formula does not hold in general for the coincidence semi-index:

$$
|\operatorname{ind}|(f, g)=0 \neq 2 \cdot 1=|\operatorname{ind}|(\bar{f}, \bar{g})|\operatorname{ind}|\left(f^{\prime}, g^{\prime}\right) .
$$

We also notice that $N(f, g)=0 \neq 1 \cdot 1=N(\bar{f}, \bar{g}) N\left(f^{\prime}, g^{\prime}\right)$.

(2.5) Lemma. Let $F, G: M \times I \rightarrow N$ be a pair of homotopies and set $f_{t}=F(\cdot, t), g_{t}=G(\cdot, t)(t \in I)$. Let $A_{0} \in \Phi^{\prime}\left(f_{0}, g_{0}\right), A_{1} \in \Phi^{\prime}\left(f_{1}, g_{1}\right)$ be two Nielsen classes corresponding under these homotopies and let $u$ be a path joining some points $x_{0} \in A_{0}, x_{1} \in A_{1}$ such that the paths $F(u(\cdot), \cdot)$, $G(u(\cdot), \cdot)$ are homotopic. Then the isomorphism $\phi: \pi_{1}\left(M, x_{0}\right) \rightarrow \pi_{1}\left(M, x_{1}\right)$ given by $\phi\langle a\rangle=\langle-u+a+u\rangle$ carries $C\left(f_{0 \#}, g_{0 \#}\right)_{x_{0}}$ onto $C\left(f_{1 \#}, g_{1 \#}\right)_{x_{1}}$ and $C^{+}\left(f_{0 \#}, g_{0 \#}\right)_{x_{0}}$ onto $C^{+}\left(f_{1 \#}, g_{1 \#}\right)_{x_{1}}$.

(2.6) Definition. A Nielsen class $A \in \Phi^{\prime}(f, g)$ is called defective if $C^{+}\left(f_{\#}, g_{\#}\right)_{x} \neq C\left(f_{\#}, g_{\#}\right)_{x} \cap f_{\#}^{-1} H^{\prime}$ for some $x \in A$.

Lemma (2.5) implies that $A$ is defective if the above inequality holds for any $x \in A$. Both (2.2) and (2.5) imply

(2.7) Corollary. Let $(f, g)$ be a pair of continuous maps and let $\left(f^{\prime}, g^{\prime}\right)$ be a transverse pair homotopic to it. Let $A \in \Phi^{\prime}(f, g)$ and $A^{\prime} \in \Phi^{\prime}\left(f^{\prime}, g^{\prime}\right)$ correspond under this homotopy. Then the class $A$ is defective iff any point of $A^{\prime}$ is self-reducing.

Recall that the Jiang group is given by $J(X, x)=\left\{a \in \pi_{1}(X, x)\right.$ : there exists a cyclic homotopy $H: \operatorname{id}_{X} \simeq \operatorname{id}_{X}$ such that $\left.\langle H(x, \cdot)\rangle=a\right\}[\mathrm{J}]$. 
(2.8) Lemma ([Y], [Je, (6.6)]). Let $f, g: M \rightarrow N$ be maps between manifolds of the same dimension and let $H \subset \pi_{1} N$ be a normal subgroup such that $H \subset J(N)$. Suppose that $A_{0}, A_{1} \in \Phi^{\prime}(f, g)$ satisfy $\operatorname{id}_{\nabla} A_{0}=\operatorname{id}_{\nabla} A_{1}$ where $\operatorname{id}_{\nabla}: \nabla(f, g) \rightarrow \nabla_{H}(f, g)$ is induced by $\left(\operatorname{id}_{M}, \operatorname{id}_{N}\right)$. Then $|\operatorname{ind}|(f, g:$ $\left.A_{0}\right)=|\operatorname{ind}|\left(f, g: A_{1}\right)$. Moreover, $A_{0}$ is defective iff $A_{1}$ is defective.

Proof. It was shown in [Je, (6.6)] that there is a cyclic homotopy $(F, G):(f, g) \simeq(f, g)$ such that $\mu_{(F, G)} A_{0}=A_{1}$. Since $\mu$ is semi-indexpreserving, the first part follows. Now the second part follows from (2.5).

(2.9) Lemma. Consider a homotopy commutative diagram

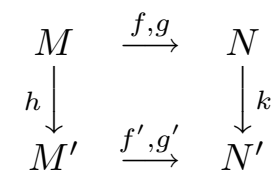

where $h$ and $k$ are homeomorphisms. Suppose that $A \in \Phi^{\prime}(f, g)$ and $\eta(A) \in$ $\Phi^{\prime}\left(f^{\prime}, g^{\prime}\right)$. Then $A \in \Phi^{\prime}(f, g)$ is defective iff $\eta(A) \in \Phi^{\prime}\left(f^{\prime}, g^{\prime}\right)$ is defective where $\eta$ is defined in Lemma (1.3)(b).

Proof. Let us represent our diagram as in the proof of (1.3):

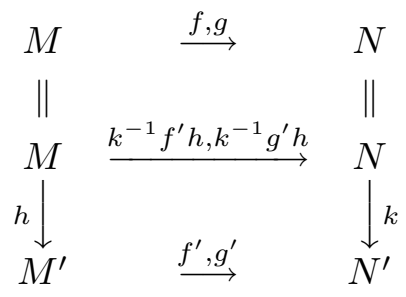

We will use the notation of that proof. Lemma (2.5) implies that $A$ is defective iff so is $\varkappa_{1} A$. Since the lower square is strictly commutative, the last is equivalent to the defectivity of $\varkappa_{2} \varkappa_{1}(A)=\eta(A)$.

3. The semi-index product formula. In this section we will show that the defective classes are the only obstructions to the coincidence semiindex product formula. To do this we will need to study the graphs of fibre maps. We first introduce the necessary notations.

Let $(V, p, E),\left(V^{\prime}, p^{\prime}, E^{\prime}\right)$ be locally trivial real $n$-dimensional vector bundles over the spaces $E$ and $E^{\prime}$ and consider a commutative diagram

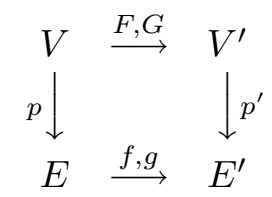


of homomorphisms of these bundles. Let $V_{x}=p^{-1}(x), V_{x^{\prime}}^{\prime}=p^{\prime-1}\left(x^{\prime}\right)$ be fibres. Denote the graphs of the restrictions by

$$
\begin{aligned}
& \Gamma_{F}(x)=\left\{(v, F v): v \in V_{x}\right\} \subset V_{x} \times V_{f x}^{\prime}, \\
& \Gamma_{G}(x)=\left\{(v, G v): v \in V_{x}\right\} \subset V_{x} \times V_{g x}^{\prime} .
\end{aligned}
$$

Then the graphs $\Gamma_{F}=\bigcup_{x \in E} \Gamma_{F}(x), \Gamma_{G}=\bigcup_{x \in E} \Gamma_{G}(x)$ are $n$-dimensional vector bundles over $E$ with projections being restrictions of the composition $V \times V^{\prime} \rightarrow V \rightarrow E$.

(3.1) Definition. We will say that $F$ is transverse to $G$ if

$$
\Gamma_{F}(x) \oplus \Gamma_{G}(x)=V_{x} \times V_{f x}^{\prime} \quad \text { for any } x \in \Phi(f, g) .
$$

(It is clear that smooth maps are transverse in the sense of Section 1 iff their tangent maps

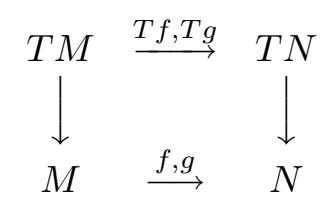

are transverse in the above sense.)

Let $V^{0} \subset V, V^{\prime 0} \subset V^{\prime}$ be $k$-dimensional subbundles such that $F\left(V^{0}\right) \cup$ $G\left(V^{0}\right) \subset V^{\prime 0}$. Let $F^{0}, G^{0}: V^{0} \rightarrow V^{\prime 0}$ denote the restrictions of $F, G$. Let $V^{1}=V / V^{0}, V^{\prime 1}=V^{\prime} / V^{\prime 0}$ denote the quotient bundles and $F^{1}, G^{1}: V^{1} \rightarrow$ $V^{\prime 1}$ the maps induced by $F, G$. (We will use this notation to study fibre maps of manifolds



Then $V=T E, V^{\prime}=T E^{\prime} ; V^{0} \subset V, V^{\prime 0} \subset V^{\prime}$ are the subbundles tangent to the fibres and $V^{1}, V^{\prime 1}$ are normal to the fibres.)

Now we have a commutative diagram with exact rows

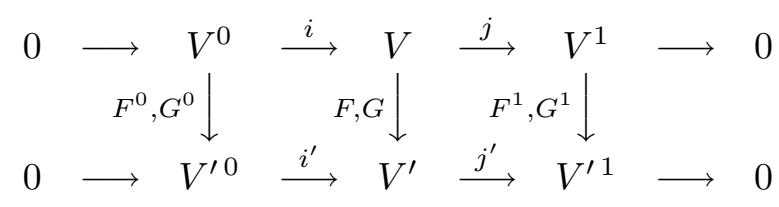

together with exact sequences of vector spaces

$$
\begin{aligned}
& 0 \rightarrow V_{x}^{0} \times V_{f x}^{\prime 0} \rightarrow V_{x} \times V_{f x}^{\prime} \rightarrow V_{x}^{1} \times V_{f x}^{\prime 1} \rightarrow 0, \\
& 0 \rightarrow V_{x}^{0} \times V_{g x}^{\prime 0} \rightarrow V_{x} \times V_{g x}^{\prime} \rightarrow V_{x}^{1} \times V_{g x}^{\prime 1} \rightarrow 0,
\end{aligned}
$$


and vector bundles

$$
\begin{aligned}
& 0 \rightarrow \Gamma_{F^{0}} \rightarrow \Gamma_{F} \rightarrow \Gamma_{F^{1}} \rightarrow 0, \\
& 0 \rightarrow \Gamma_{G^{0}} \rightarrow \Gamma_{G} \rightarrow \Gamma_{G^{1}} \rightarrow 0 .
\end{aligned}
$$

One can easily check

(3.2) Corollary. If any two pairs out of $\left(F^{0}, G^{0}\right),(F, G),\left(F^{1}, G^{1}\right)$ are transverse then so is the third.

Let $x, y \in \Phi(f, g)$ and let a path $u$ establish the Nielsen relation between them. Denote by $\delta_{t}(F)\left(\delta_{t}(G)\right)$ the translation of the orientation of the bundle $\Gamma_{F}\left(\Gamma_{G}\right)$ along $u$. Then $\delta_{0}=\delta_{0}(F) \wedge \delta_{0}(G)$ is an orientation of $V_{x} \times V_{f x}^{\prime}$; let $\delta_{1}$ be its translation in the fibre bundle $p \times p^{\prime}: V \times V^{\prime} \rightarrow E \times E^{\prime}$ along the path $(u, f u)$ (or equivalently along $(u, g u)$ ).

(3.3) Definition. We say that the path $u$ is graph-orientation-preserving (-reversing) if $\delta_{1}=\delta_{1}(F) \wedge \delta_{1}(G)\left(\delta_{1}=-\delta_{1}(F) \wedge \delta_{1}(G)\right)$.

Consider again a transverse pair $f, g: M \rightarrow N$ and a path $u$ establishing the Nielsen relation between two coincidence points. Then $u$ is graphorientation-preserving in the sense of Section 1 iff it is so in the above sense for

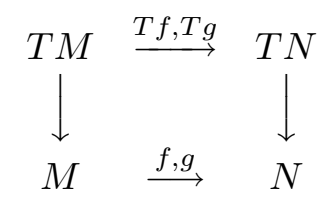

(3.4) Definition. Let $0 \rightarrow V^{0} \rightarrow V \stackrel{j}{\rightarrow} V^{1} \rightarrow 0$ be a short exact sequence of finite-dimensional vector spaces. Orientations $\alpha^{0}, \alpha, \alpha^{1}$ of these spaces will be called compatible if there exists an ordered basis $\left(a_{1}, \ldots, a_{n}\right)$ of $V$ such that $\alpha^{0}=\left[\left(a_{1}, \ldots, a_{s}\right)\right], \alpha=\left[\left(a_{1}, \ldots, a_{n}\right)\right], \alpha^{1}=\left[\left(j a_{s+1}, \ldots, j a_{n}\right)\right]$ (here $\left.s=\operatorname{dim} V^{0}\right)$.

(3.5) Re mark. Let $0 \rightarrow V^{0} \rightarrow V \rightarrow V^{1} \rightarrow 0$ be an exact sequence of locally trivial vector bundles over a space $E$. Let $u$ be a path in $E$ and let $\alpha_{t}^{0}, \alpha_{t}, \alpha_{t}^{1}$ be translations of some orientations along $u$. Then $\alpha_{0}^{0}, \alpha_{0}, \alpha_{0}^{1}$ are compatible iff $\alpha_{t}^{0}, \alpha_{t}, \alpha_{t}^{1}$ are compatible for any $t \in[0,1]$.

(3.6) LEMMA. Consider a commutative diagram of vector bundles

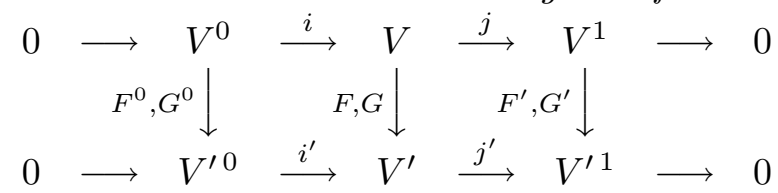

where $i, j$ and $i^{\prime}, j^{\prime}$ cover the identity maps of $E$ and $E^{\prime}$ respectively, the rows are exact and $\left(F^{0}, G^{0}\right),(F, G),\left(F^{1}, G^{1}\right)$ are transverse pairs of vector bundle homomorphisms covering some maps $f, g: E \rightarrow E^{\prime}$. Let a path 
$u$ establish the Nielsen relation between some $x, y \in \Phi(f, g)$. Then if $u$ is graph-orientation-preserving with respect to two pairs out of $\left(F^{0}, G^{0}\right)$, $(F, G),\left(F^{1}, G^{1}\right)$ then it is so with respect to the third.

$\operatorname{Proof}$. Put $\varepsilon=+1(-1)$ if $u$ is graph-orientation-preserving (-reversing) with respect to $(F, G)$. Similarly we define $\varepsilon^{0}$ and $\varepsilon^{1}$. We will show that $\varepsilon=\varepsilon^{0} \varepsilon^{1}$.

Denote by $\alpha_{t}\left(F^{0}\right), \alpha_{t}(F), \alpha_{t}\left(F^{1}\right)$ and $\alpha_{t}\left(G^{0}\right), \alpha_{t}(G), \alpha_{t}\left(G^{1}\right)$ compatible translations of orientations in

$$
0 \rightarrow \Gamma_{F^{0}} \rightarrow \Gamma_{F} \rightarrow \Gamma_{F^{1}} \rightarrow 0 \quad \text { and } \quad 0 \rightarrow \Gamma_{G^{0}} \rightarrow \Gamma_{G} \rightarrow \Gamma_{G^{1}} \rightarrow 0
$$

along $u$. Set $\alpha_{0}^{0}=\alpha_{0}\left(F^{0}\right) \wedge \alpha_{0}\left(G^{0}\right), \alpha_{0}=\alpha_{0}(F) \wedge \alpha_{0}(G)$ and $\alpha_{0}^{1}=\alpha_{0}\left(F^{1}\right) \wedge$ $\alpha_{0}\left(G^{1}\right)$. Then $\alpha_{0}^{0},(-1)^{k l} \alpha_{0}, \alpha_{0}^{1}$ are compatible (here $k=\operatorname{dim} E_{x}^{0}, l=$ $\left.\operatorname{dim} E_{x}^{1}\right)$. Let $\alpha_{t}^{0},(-1)^{k l} \alpha_{t}, \alpha_{t}^{1}$ be the translations in the bundles $V^{0} \times V^{\prime 0}$, $V \times V^{\prime}, V^{1} \times V^{\prime 1} \rightarrow E \times E^{\prime}$ along $(u, f u)$. By (3.5) we get for $t=1$ the compatible orientations

$$
\alpha_{1}^{0},(-1)^{k l} \alpha_{1}, \alpha_{1}^{1}
$$

But

$$
\left\{\begin{array}{l}
\alpha_{1}^{0}=\varepsilon^{0} \alpha_{1}\left(F^{0}\right) \wedge \alpha_{1}\left(G^{0}\right), \\
\alpha_{1}=\varepsilon \alpha_{1}(F) \wedge \alpha_{1}(G), \\
\alpha_{1}^{1}=\varepsilon^{1} \alpha_{1}\left(F^{1}\right) \wedge \alpha_{1}\left(G^{1}\right) .
\end{array}\right.
$$

Now since $\alpha_{1}\left(F^{0}\right), \alpha_{1}(F), \alpha_{1}\left(F^{1}\right)$ and $\alpha_{1}\left(G^{0}\right), \alpha_{1}(G), \alpha_{1}\left(G^{1}\right)$ are compatible, so are also

$$
(* * *) \quad \alpha_{1}\left(F^{0}\right) \wedge \alpha_{1}\left(G^{0}\right),(-1)^{k l} \alpha_{1}(F) \wedge \alpha_{1}(G), \alpha_{1}\left(F^{1}\right) \wedge \alpha_{1}\left(G^{1}\right) .
$$

Finally, we substitute $(* *)$ into $(*)$ and compare with $(* * *)$. This implies $\varepsilon^{0} \varepsilon \varepsilon^{1}=1$.

(3.7) Lemma. Consider a commutative diagram of homomorphisms between $k$-dimensional vector bundles

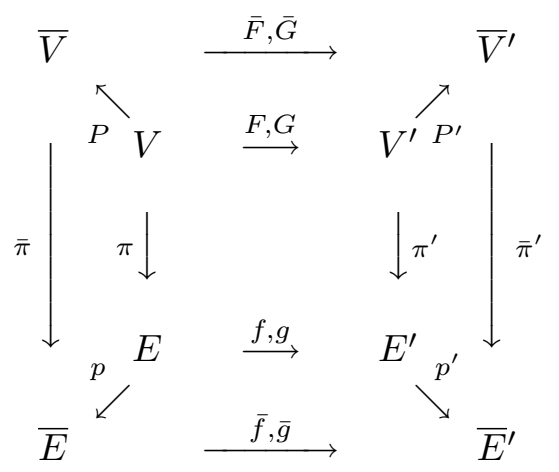


Let $(F, G),(\bar{F}, \bar{G})$ be transverse and let $P, P^{\prime}$ be isomorphisms on the fibres. Let $x, y \in \Phi(f, g)$ and let $u$ establish the Nielsen relation between them. Then $\bar{x}=p x, \bar{y}=p y \in \Phi(\bar{f}, \bar{g})$ and $\bar{u}=p u$ establishes the Nielsen relation. Moreover, $u$ is graph-orientation-preserving iff so is $\bar{u}$.

Proof. Only the last statement requires a proof. Let $\delta_{t}(F)$ and $\delta_{t}(G)$ denote orientations of $\Gamma_{u(t)}(F)$ and $\Gamma_{u(t)}(G)$ respectively. Then $\delta_{t}(\bar{F})=$ $\left(P \times P^{\prime}\right)_{*} \delta_{t}(F), \delta_{t}(\bar{G})=\left(P \times P^{\prime}\right)_{*} \delta_{t}(G)$ are orientations of $\Gamma_{\bar{u}(t)}(\bar{F})$ and $\Gamma_{\bar{u}(t)}(\bar{G})$ respectively. Then $\delta_{0}=\delta_{0}(F) \wedge \delta_{0}(\bar{G})$ is an orientation of $V_{x} \times$ $V_{f x}^{\prime}$. Let $\bar{\delta}_{t}$ be its translation along $(u, f u)$. Then $\bar{\delta}_{0}=\left(P \times P^{\prime}\right)_{*} \delta_{0}$ is an orientation of $V_{x} \times V_{f x}^{\prime}$ and $\bar{\delta}_{t}=\left(P \times P^{\prime}\right)_{*} \delta_{t}$ is its translation along $(\bar{u}, f \bar{u})$. Suppose that $\delta_{1}=\varepsilon \delta_{1}(F) \wedge \delta_{1}(G)$. Then $\bar{\delta}_{1}=\left(P \times P^{\prime}\right)_{*} \delta_{1}=$ $\varepsilon\left[\left(P \times P^{\prime}\right)_{*} \delta_{1}(F)\right] \wedge\left[\left(P \times P^{\prime}\right)_{*} \delta_{1}(G)\right]=\varepsilon \delta_{1}(\bar{F}) \wedge \delta_{1}(\bar{G})$.

Consider a locally trivial fibre bundle $(E, p, B)$ where all spaces involved are smooth manifolds. Set

$$
\begin{aligned}
T E & =\text { the tangent bundle to } E \\
T^{0} E & =\text { the bundle tangent to the fibres, } \\
\nu(E) & =T E / T^{0} E=\text { the bundle normal to the fibres. }
\end{aligned}
$$

Then $p: E \rightarrow B$ induces the vector bundle homomorphism

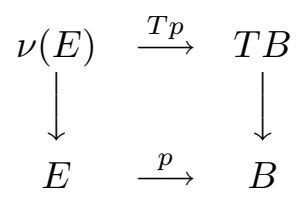

which is an isomorphism on the fibres.

(3.9) Lemma. Consider a commutative diagram

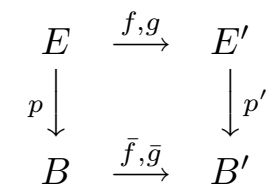

and suppose that the pairs $(f, g),(\bar{f}, \bar{g})$ and $\left(f_{b}, g_{b}\right)$ are transverse for any $b \in \Phi(\bar{f}, \bar{g})$. Let $x_{0}, x_{1} \in \Phi(f, g)$ and let $u$ be a path establishing the Nielsen relation between them. Consider:

(a) the diagram

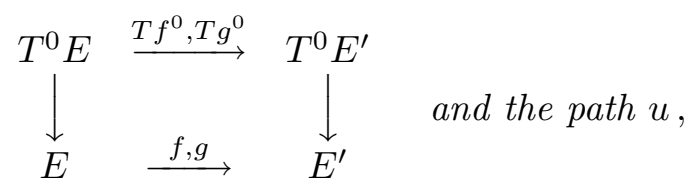


(b) the diagram

$$
\stackrel{T E}{\downarrow} \stackrel{T f, T g}{\longrightarrow} T E^{\prime}
$$

(c) the diagram

$$
\underset{\downarrow}{T B} \stackrel{T \bar{f}, T \bar{g}}{\longrightarrow} T B^{\prime}
$$

If in any two of the above cases the path considered is graph-orientationpreserving, then the same is true in the third case.

Pr o of. We consider the commutative diagram with exact rows

$$
\begin{aligned}
& 0 \longrightarrow T^{0} E \longrightarrow T E \longrightarrow \nu(E) \longrightarrow 0 \\
& T f^{0}, T g^{0} \downarrow \quad T f, T g \downarrow \quad T f^{1}, T g^{1} \downarrow \\
& 0 \longrightarrow T^{0} E^{\prime} \longrightarrow T E^{\prime} \longrightarrow \nu\left(E^{\prime}\right) \longrightarrow 0
\end{aligned}
$$

By (3.6), $u$ is graph-orientation-preserving with respect to $(T f, T g)$ if it is simultaneously orientation-preserving or orientation-reversing with respect to $\left(T f^{0}, T g^{0}\right)$ and $\left(T f^{1}, T g^{1}\right)$. But by (3.7), $u$ is orientation-preserving with respect to $\left(T f^{1}, T g^{1}\right)$ iff so is $\bar{u}$ with respect to $(T \bar{f}, T \bar{g})$.

(3.10) Lemma. Consider a homomorphism of vector bundles

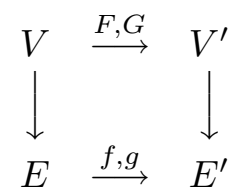

Let $F, G$ be transverse and let $u$ be a path in $E$ satisfying $f u=g u$. Then $u$ is graph-orientation-preserving.

Proof. For any $t \in[0,1]$ we have

$$
\Gamma_{F}(u(t)) \oplus \Gamma_{G}(u(t))=V_{u(t)} \times V_{f u(t)}^{\prime} .
$$

If $\alpha_{t}(F)$ and $\alpha_{t}(G)$ are translations of some orientations of $\Gamma_{F}$ and $\Gamma_{G}$ along $u$ then $\alpha_{t}(F) \wedge \alpha_{t}(G)$ is the translation of an orientation of the bundle $V \times V^{\prime} \rightarrow E \times E^{\prime}$ along $(u, f u=g u)$. 
(3.11) Lemma. Consider a commutative diagram

$(*)$

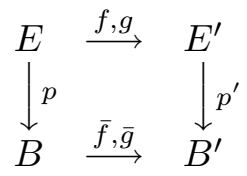

where $(E, p, B),\left(E^{\prime}, p^{\prime}, B^{\prime}\right)$ are locally trivial fibre bundles whose fibres are smooth closed n-manifolds. Suppose that $f_{b}, g_{b}$ are transverse for $b \in \Phi(\bar{f}, \bar{g})$. Let $b_{0}, b_{1} \in \Phi(\bar{f}, \bar{g})$ and let $\bar{u}$ establish the Nielsen relation between $b_{0}$ and $b_{1}$. Then there exist decompositions (maybe incomplete) $\Phi\left(f_{b_{0}}, g_{b_{0}}\right)=A_{0} \cup$ $\left\{w_{1}, \ldots, w_{r}\right\}, \Phi\left(f_{b_{1}}, g_{b_{1}}\right)=A_{1} \cup\left\{w_{1}^{\prime}, \ldots, w_{r}^{\prime}\right\}$ and paths $u_{i}$ in $E$ joining $w_{i}$ and $w_{i}^{\prime}$ and such that $f u_{i} \simeq g u_{i}, p u_{i} \simeq \bar{u}$ and $u_{i}$ is graph-orientationpreserving with respect to $T f^{0}, T g^{0}: T^{0} E \rightarrow T^{0} E^{\prime}(i=1, \ldots, r)$.

Pr o of. Let $\bar{H}$ be a homotopy between $\bar{f} \bar{u}$ and $\bar{g} \bar{u}$. Let $K=I \times I / \sim$ be the quotient space obtained by identifying $0 \times I$ and $1 \times I$ to single points. Then $\bar{H}$ defines a map $\bar{H}: K \rightarrow B^{\prime}$. We consider the bundles induced by $\bar{u}$ and $\bar{H}$ :

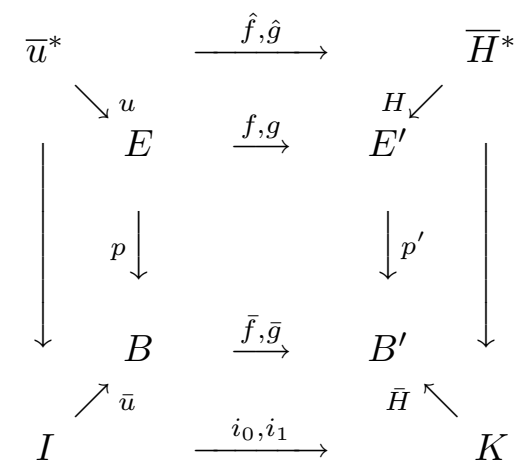

where

$$
\begin{gathered}
\bar{u}^{*}=\{(e, t) \in E \times I: p(e)=\bar{u}(t)\}, \\
\bar{H}^{*}=\left\{\left(e^{\prime},[t, s]\right) \in E^{\prime} \times K: p^{\prime}\left(e^{\prime}\right)=\bar{H}(t, s)\right\}, \\
i_{0}(t)=[t, 0], \quad i_{1}(t)=[t, 1] .
\end{gathered}
$$

First we will prove the lemma for the induced diagram, $b_{0}=0, b_{1}=1$ and $u=$ identity on $I$. Since bundles over contractible spaces are trivial, we get the diagram



where $V=E_{b}, V^{\prime}=E_{\bar{f} b}^{\prime}$. 
Now we may write $\widehat{f}(v, t)=\left(f_{1}(v, t),[t, 0]\right), \widehat{g}(v, t)=\left(g_{1}(v, t),[t, 1]\right)$ and notice that the pairs $\left(f_{1}(\cdot, 0), g_{1}(\cdot, 0)\right)$ and $\left(f_{1}(\cdot, 1), g_{1}(\cdot, 1)\right)$ are transverse. The maps $f_{1}, g_{1}: V \times I \rightarrow V^{\prime}$ are homotopic rel $V \times\{0,1\}$ to a transverse pair and so we assume they are transverse. Then $\Phi\left(f_{1}, g_{1}\right)$ is a 1-manifold and we get

$$
\begin{aligned}
& \Phi\left(f_{1}(\cdot, 0), g_{1}(\cdot, 0)\right)=\left\{x_{1}, y_{1}, \ldots, x_{k}, y_{k}: w_{1}, \ldots, w_{r}\right\}, \\
& \Phi\left(f_{1}(\cdot, 1), g_{1}(\cdot, 1)\right)=\left\{x_{1}^{\prime}, y_{1}^{\prime}, \ldots, x_{l}^{\prime}, y_{l}^{\prime}: w_{1}^{\prime}, \ldots, w_{r}^{\prime}\right\},
\end{aligned}
$$

where $\left\{x_{i}, y_{i}\right\},\left\{x_{i}^{\prime}, y_{i}^{\prime}\right\},\left\{w_{i}, w_{i}^{\prime}\right\}$ are the ends of connected components of $\Phi\left(f_{1}, g_{1}\right)$. The proof of (1.4) in [DJ] implies that $x_{i} R y_{i}$ as coincidence points of $\left(f_{1}(\cdot, 0), g_{1}(\cdot, 0)\right)$ and $x_{i}^{\prime} R y_{i}^{\prime}$ as coincidence points of $\left(f_{1}(\cdot, 1), g_{1}(\cdot, 1)\right)$, which proves the first part of lemma for the induced diagrams.

Now consider the points $w_{i}, w_{i}^{\prime}$. Let $u=\left(u_{1}, u_{2}\right): I \rightarrow V \times I$ denote the component joining them. Then $f_{1} u(t)=g_{1} u(t)$ and hence the paths $f u$ and $g u$ are homotopic in $V^{\prime} \times K$. Now we will show that $u$ is graph-orientationpreserving on the fibre. We consider the diagram

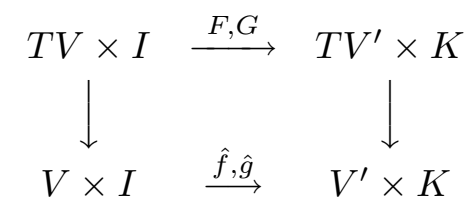

(here $\left.F(v, t)=\left(T f_{1(x, t)}(v),[t, 0]\right), G(v, t)=\left(T g_{1(x, t)}(v),[t, 1]\right), v \in T_{x} V\right)$ and the family of diagrams

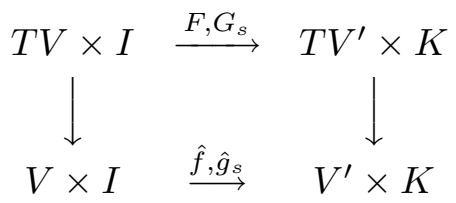

(here $\left.\widehat{g}_{s}(v, t)=\left(g_{1}(v, t),[t, s]\right), G_{s}(v, t)=\left(T g_{1(x, t)}(v),[t, s]\right), v \in T_{x} V\right)$. Then $\left(F, G_{s}\right)$ is transverse for any $s \in I$. Since $G_{1}=G$, it remains to show that $u$ is graph-orientation-preserving for $\left(F, G_{0}\right)$. But $f u=g_{0} u$ and we apply (3.10). This ends the proof in the special case of the induced diagram. To prove the general case we notice that the diagram $(* *)$ is induced from (*) and we apply (3.7).

For the remainder of this section we will consider a commutative diagram

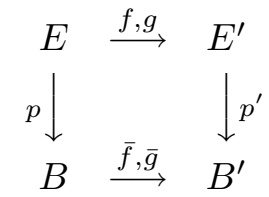


of locally trivial fibre bundles where all spaces involved are smooth closed connected manifolds of respectively equal dimensions.

(3.12) Lemma. Let $(f, g)$ and $(\bar{f}, \bar{g})$ be transverse and let $A \in \Phi^{\prime}(f, g)$, $\bar{A} \in \Phi^{\prime}(\bar{f}, \bar{g})$ satisfy $p A \subseteq \bar{A}$. Let $\bar{A}=\bar{A}_{0} \cup\left\{b_{1}, \ldots, b_{m}\right\}$ be a decomposition. Then

(i) $A \cap p^{-1}\left(\bar{A}_{0}\right) \subset \Phi(f, g)$ may be written as a set of pairs of $R$-related points.

Now fix decompositions $A \cap E_{b_{i}}=A_{i} \cup\left\{z_{1}^{i}, \ldots, z_{s}^{i}\right\}$ with respect to $\left(f_{b_{i}}, g_{b_{i}}\right)$. Then

(ii) any path $\bar{u}$ from $b_{i}$ to $b_{j}$ satisfying $\bar{f} \bar{u} \simeq \overline{g u}$ gives rise to a bijective map $\phi:\left(z_{1}^{i}, \ldots, z_{s}^{i}\right) \rightarrow\left(z_{1}^{j}, \ldots, z_{s}^{j}\right)$ such that there exist paths $u_{k}$ from $z_{k}^{i}$ to $\phi\left(z_{k}^{i}\right)$ satisfying $f u_{k} \simeq g u_{k}, p u_{k} \simeq \bar{u}$ and $u_{k}$ is graph-orientation-preserving on the fibre $(i, j=1, \ldots, m ; k=1, \ldots, s)$.

Proof. Let $\bar{A}=\bar{A}_{0} \cup\left\{b_{1}, \ldots, b_{m}\right\}=\left\{a_{1}, a_{1}^{\prime}, \ldots, a_{k}, a_{k}^{\prime}: b_{1}, \ldots, b_{m}\right\}$. First we will prove that $p^{-1}\left\{a_{i}, a_{i}^{\prime}\right\}$ splits into pairs of $R$-related points. Let $\bar{u}$ be a graph-orientation-reversing path establishing the Nielsen relation between $a_{i}$ and $a_{i}^{\prime}$. Lemma (3.11) gives us decompositions

$$
\begin{aligned}
& A \cap E_{a_{i}}=\left\{x_{1}, y_{1}, \ldots, x_{k}, y_{k}: w_{1}, \ldots, w_{r}\right\}, \\
& A \cap E_{a_{i}^{\prime}}=\left\{x_{1}^{\prime}, y_{1}^{\prime}, \ldots, x_{l}^{\prime}, y_{l}^{\prime}: w_{1}^{\prime}, \ldots, w_{r}^{\prime}\right\} .
\end{aligned}
$$

It remains to show that $w_{i} R w_{i}^{\prime}$. Lemma (3.11) gives us a path $u_{i}$ from $w_{i}$ to $w_{i}^{\prime}$ satisfying $p u_{i} \simeq \bar{u}, f u_{i} \simeq g u_{i}$ and graph-orientation-preserving on the fibre. Now by (3.9), $u_{i}$ is graph-orientation-reversing on the total space, which implies $w_{i} R w_{i}^{\prime}$.

Now we prove the second part.

Fix $i, j=1, \ldots, m$ and a path $\bar{u}$ from $b_{i}$ to $b_{j}$ satisfying $\bar{f} \bar{u} \simeq \bar{g} \bar{u}$. Then (3.11) gives us (incomplete) decompositions $A \cap E_{b_{i}}=A_{i}^{\prime} \cup\left\{z_{1}^{\prime i}, \ldots, z_{t}^{\prime i}\right\}, A \cap$ $E_{b_{j}}=A_{j}^{\prime} \cup\left\{z_{1}^{\prime j}, \ldots, z_{t}^{\prime j}\right\}$ and paths $u_{k}^{\prime}$ from $z_{k}^{\prime i}$ to $z_{k}^{\prime j}$ such that $f u_{k}^{\prime} \simeq g u_{k}^{\prime}$, $p u_{k}^{\prime} \simeq \bar{u}$ and $u_{k}^{\prime}$ is graph-orientation-preserving on the fibre. Suppose now that $A \cap E_{b_{i}}=A_{i}^{\prime \prime} \cup\left\{z_{1}^{\prime i}, \ldots, z_{s}^{\prime i}\right\}$ is a complete decomposition $(s \leq t)$. Then by (3.11) the elements $\left\{z_{1}^{\prime j}, \ldots, z_{s}^{\prime j}\right\}$ are also free in a decomposition $A \cap$ $E_{b_{j}}=A_{j}^{\prime \prime} \cup\left\{z_{1}^{\prime j}, \ldots, z_{s}^{\prime j}\right\}$. Applying Lemma (1.4) in [DJ] to $f_{b_{i}}, g_{b_{i}}: E_{b_{i}} \rightarrow$ $E_{\bar{f} b_{i}}^{\prime}$ gives us a bijective map between the sets $\left\{z_{1}^{i}, \ldots, z_{s}^{i}\right\}$ and $\left\{z_{1}^{\prime i}, \ldots, z_{s}^{\prime i}\right\}$ (we may assume that $z_{k}^{i}$ corresponds to $z_{k}^{\prime i}$ ) and paths $v_{k}$ joining these points in $E_{b_{i}}$ satisfying $f v_{k} \simeq g v_{k}$ and graph-orientation-preserving.

Then we consider the maps $f_{b_{j}}, g_{b_{j}}: E_{b_{j}} \rightarrow E_{\bar{f} b_{j}}^{\prime}$ and we find paths $w_{k}$ joining $z_{k}^{j}$ and $z_{k}^{\prime j}$ in $E_{b_{j}}$ with similar properties. Finally, the composition $u_{k}=v_{k}+u_{k}^{\prime}-w_{k}$ satisfies the conditions of our lemma.

Now we are in a position to prove the main result of this section. 
(3.13) Theorem. Consider a diagram

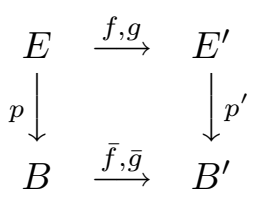

and let $A \in \Phi^{\prime}(f, g), p A \subseteq \bar{A} \in \Phi^{\prime}(\bar{f}, \bar{g})$. Then

(i) for any $b \in \bar{A}$

$$
|\operatorname{ind}|(f, g: A) \leq|\operatorname{ind}|(\bar{f}, \bar{g}: \bar{A})|\operatorname{ind}|\left(f_{b}, g_{b}: A \cap E_{b}\right),
$$

(ii) (semi-index product formula, SIPF) the equality

$$
|\operatorname{ind}|(f, g: A)=|\operatorname{ind}|(\bar{f}, \bar{g}: \bar{A})|\operatorname{ind}|\left(f_{b}, g_{b}: A \cap E_{b}\right)
$$

holds iff at least one of the following conditions is satisfied:

(a) $|\operatorname{ind}|(\bar{f}, \bar{g}: \bar{A})|\operatorname{ind}|\left(f_{b}, g_{b}: A \cap E_{b}\right) \leq 1$,

(b) the class $\bar{A}$ is not defective and neither is any class in $A \cap E_{b}$.

Proof. Let $\bar{A}=\bar{A}_{0} \cup\left\{b_{1}, \ldots, b_{m}\right\}, E_{b_{i}} \cap A=A_{i} \cup\left\{z_{1}^{i}, \ldots, z_{s}^{i}\right\}(i=$ $1, \ldots, m)$ be complete decompositions. Then by (3.12), $A-\left\{z_{j}^{i}: i=\right.$ $1, \ldots, m ; j=1, \ldots, s\}$ splits into pairs of $R$-related points. So $\mid$ ind $\mid(f, g: A)$ $\leq \#\left\{z_{j}^{i}: i=1, \ldots, m ; j=1, \ldots, s\right\}=m s=|\operatorname{ind}|(\bar{f}, \bar{g}: \bar{A})|\operatorname{ind}|\left(f_{b}, g_{b}:\right.$ $\left.E_{b} \cap A\right)$ and equality holds iff no two different points from $\left\{z_{j}^{i}\right\}$ are $R$ related.

(a) If $m s=0$ then there are no free points. If $m=s=1$ then there is exactly one free point and hence also $\mid$ ind $\mid(f, g: A)=1$.

(b) Assume that (b) holds and $z_{k}^{i} R z_{k^{\prime}}^{i \prime}$. This gives us a path $u^{\prime \prime}$ between these points satisfying $f u^{\prime \prime} \simeq g u^{\prime \prime}$ and graph-orientation-reversing. Since there are no self-reducing points in $A$ and $b_{i}, b_{i^{\prime}}$ are free, $u^{\prime \prime}$ is graphorientation-preserving on the fibre. On the other hand, (3.12) gives a path $u^{\prime}$ from $z_{k^{\prime}}^{i \prime}$ to some $z_{k^{\prime \prime}}^{i}$ satisfying $f u^{\prime} \simeq g u^{\prime}$ and graph-orientation-preserving on the fibre. We may also assume that $p u^{\prime} \simeq p u^{\prime \prime}$, so $p\left(u^{\prime}-u^{\prime \prime}\right)$ is null homotopic and hence $u^{\prime}-u^{\prime \prime}$ is homotopic to a path lying in $E_{b_{i}}$. This path is also graph-orientation-reversing on the fibre and thus $z_{k}^{i} R z_{k^{\prime \prime}}^{i}$ as coincidence points of $\left(f_{b_{i}}, g_{b_{i}}\right)$. If $k \neq k^{\prime \prime}$ we get two free $R$-related points and if $k=k^{\prime \prime}$ a self-reducing point of $\left(f_{b_{i}}, g_{b_{i}}\right)$. In any case we get a contradiction.

Now we assume that SIPF holds, i.e. no two distinct points from $\left\{z_{j}^{i}\right\}$ are $R$-related. We will show that if (b) does not hold then the class $A$ is defective. First suppose that $x R x$ for some $x \in A \cap E_{b} \subset \Phi\left(f_{b}, g_{b}\right)$. Then $x R x$ as a coincidence point of $(f, g)$ and $A \subset \Phi(f, g)$ is defective. Now assume that $b R b$ for some $b \in p A \subset \Phi(\bar{f}, \bar{g})$. Then a loop $\bar{u}$ based at $b$ satisfies $\bar{f} \bar{u} \simeq \bar{g} \bar{u}$ and is graph-orientation-reversing. We apply (3.12) to $b=b_{i}=b_{j}$ and we get a path $u$ joining some free points $z_{k}, z_{l} \in A \cap E_{b}$, 
graph-orientation-preserving on the fibre and such that $p u \simeq \bar{u}$. Then, by (3.10), $u$ is graph-orientation-reversing on the total spaces. Then if $k \neq l$ then $z_{k} R z_{l}$ as coincidence points of $(f, g)$, contradicting SIPF. Thus $k=l$ and the class $A \subset \Phi(f, g)$ is defective.

In any case $A$ is defective and it is enough to notice that for a defective class SIPF implies (a).

4. The Nielsen number product formula. Consider a commutative diagram

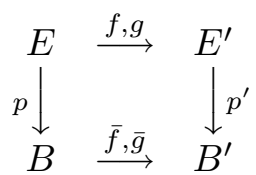

where all spaces involved are smooth closed connected manifolds of respectively equal dimensions. This diagram induces a map $p_{\nabla}: \nabla(f, g) \rightarrow \nabla(\bar{f}, \bar{g})$ and similarly for any $b \in \Phi(\bar{f}, \bar{g})$ the diagram

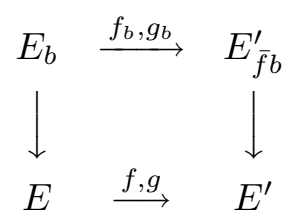

(the vertical arrows are inclusions) induces $\left(i_{b}\right)_{\nabla}: \nabla_{K}\left(f_{b}, g_{b}\right) \rightarrow \nabla(f, g)$ (see $[\mathrm{Y}]$ or $[\mathrm{Je}])$. Recall also that the transformation $T$ from the end of Section 1 determines the action of the group $C\left(\bar{f}_{\#}, \bar{g}_{\#}\right)_{b}$ on $\nabla_{K}\left(f_{b}, g_{b}\right)(b \in$ $\Phi(\bar{f}, \bar{g}))$. It turns out that the orbits of the above action coincide with the counterimages $\left(i_{b}\right)_{\nabla}^{-1} A(A \in \nabla(f, g), b \in p A)$ (see [Je, (4.11)]). Since $T$ is semi-index-preserving, $\mid$ ind $\left|\left(f_{b}, g_{b}: A_{0}\right)=\right|$ ind $\mid\left(f_{b}, g_{b}: A_{1}\right)$ for $A_{0}, A_{1} \in$ $\left(i_{b}\right)_{\nabla}^{-1} A$.

(4.2) Lemma. Let $A \in \Phi^{\prime}(f, g)$ and $b \in p A$. If $A$ is essential then so is $p_{\nabla} A \in \Phi^{\prime}(\bar{f}, \bar{g})$ and any class in the orbit $\left(i_{b}\right)_{\nabla}^{-1} A$. If $A$ satisfies SIPF then the converse is also true.

Proof. The first part follows from the inequality in (3.13)(i) and the fact that $|\operatorname{ind}|\left(f_{b}, g_{b}: E_{b} \cap A\right) \neq 0$ iff $\left(i_{b}\right)_{\nabla}^{-1} A$ is an essential orbit (which follows from the additivity of the semi-index (see [DJ, (1.11d)])). The second part is evident.

Example (2.4) shows that the converse is not true in general.

Now assume that all the Nielsen classes satisfy SIPF. Let $\bar{A}_{1}, \ldots, \bar{A}_{s}$ be all the essential classes of $(\bar{f}, \bar{g})$. Now (4.2) implies that if $A \in \nabla(f, g)$ is essential then so is $p A \in \Phi^{\prime}(\bar{f}, \bar{g})$. Hence $p A=\bar{A}_{i}$ for some $i=1, \ldots, s$. Set

$$
C_{i}=\#\left\{A \in \Phi^{\prime}(f, g): \mid \text { ind } \mid(f, g: A) \neq 0, p A=\bar{A}_{i}\right\} .
$$


Then $N(f, g)=C_{1}+\ldots+C_{s}, N(\bar{f}, \bar{g})=s$.

Let $b_{i} \in \bar{A}_{i}$. Then SIPF implies that any essential orbit in $\nabla_{K}\left(f_{b_{i}}, g_{b_{i}}\right)$ is of the form $\left(i_{b_{i}}\right)_{\nabla}^{-1} A$ for an essential class $A \in \Phi^{\prime}(f, g)$. Thus $C_{i}$ equals the number of these orbits. On the other hand (see [Je, (6.3), (6.2)]), the length of the orbit $\left(i_{b_{i}}\right)_{\nabla}^{-1} A$ equals the index of the subgroup $p_{\#}\left(C\left(f_{\#}, g_{\#}\right)_{x}\right)$ in the group $C\left(\bar{f}_{\#}, \bar{g}_{\#}\right)_{b}\left(x \in E_{b} \cap A\right)$. Thus we get

(4.3) Theorem. Assume that in the diagram (4.1) all the classes of $(f, g)$ satisfy SIPF and $N(\bar{f}, \bar{g}) \neq 0$. Choose a point in each essential class, $b_{i} \in \bar{A}_{i} \in \Phi(\bar{f}, \bar{g}), i=1, \ldots, s$. Then $N(f, g)=\sum_{i=1}^{s} N\left(f_{b_{i}}, g_{b_{i}}\right)$ iff the following two conditions are satisfied:

(a) $N_{K}\left(f_{b_{i}}, g_{b_{i}}\right)=N\left(f_{b_{i}}, g_{b_{i}}\right), i=1, \ldots, s$

(b) $C\left(\bar{f}_{\#}, \bar{g}_{\#}\right)_{b}=p_{\#} C\left(f_{\#}, g_{\#}\right)_{x}$ for any $x$ lying in an essential class of $(f, g), b=p x$.

Proof. As we have mentioned $N(f, g)=C_{1}+\ldots+C_{s}$. But $C_{i} \leq$ $N_{K}\left(f_{b_{i}}, g_{b_{i}}\right) \leq N\left(f_{b_{i}}, g_{b_{i}}\right)$ and it is easy to see that $C_{i}=N_{K}\left(f_{b_{i}}, g_{b_{i}}\right)$ iff any essential orbit consists of one element; but this is equivalent to (b).

The above theorem may be regarded as a generalization of $[\mathrm{Y},(5.6)]$ and $[\mathrm{Je},(6.5)]$. In fact, if we add the assumption that $\left(E^{\prime}, p^{\prime}, B^{\prime}\right)$ is orientable in the sense of You ([Y, after (5.5)]) then $N\left(f_{b_{i}}, g_{b_{i}}\right)$ does not depend on $i$ and

$$
\sum_{i=1}^{s} N\left(f_{b_{i}}, g_{b_{i}}\right)=s N\left(f_{b}, g_{b}\right)=N(\bar{f}, \bar{g}) N\left(f_{b}, g_{b}\right) .
$$

(4.4) Corollary. If in the diagram (4.1), $B=B^{\prime}=T^{n}$ is the $n$ dimensional torus, then the formula of (4.3) holds for any fibre map.

Proof. Let $n \times n$ integer matrices $A$ and $B$ represent the homotopy group homomorphisms induced by the maps $\bar{f}, \bar{g}: T^{n} \rightarrow T^{n}$. Assume first that $\operatorname{det}(A-B)=0$. Then $(\bar{f}, \bar{g})$ is homotopic to a coincidence free pair ([Je, (7.3)(a)]), hence so is $(f, g)$ and $N(f, g)=N(\bar{f}, \bar{g})=0$. Now suppose that $\operatorname{det}(A-B) \neq 0$. We will show that the assumptions of (4.3) are satisfied. We notice that the index of any Reidemeister class of $(\bar{f}, \bar{g})$ equals \pm 1 (see the proof of $[\mathrm{Je},(7.3)])$. It was also shown ([Je, (7.6)]) that $C\left(f_{\#}, g_{\#}\right)=0$ in this case. On the other hand, the homotopy exact sequence of $\left(E^{\prime}, p^{\prime}, T^{n}\right)$ shows that $K=0$. Thus it remains to show that any class $A \in \Phi^{\prime}(f, g)$ satisfies SIPF.

We consider two cases: first we assume that no defective class of $\left(f_{b}, g_{b}\right)$ is contained in $A(b \in p A)$. Since $T^{n}$ is orientable, no class of $(\bar{f}, \bar{g})$ is defective, hence the assumption (b) of (3.13) is satisfied and SIPF follows. Now assume that $A \cap E_{b}$ contains a self-reducing point. In general $A \cap E_{b}$ is the sum of the classes contained in one orbit of the action of $C\left(\bar{f}_{\#}, \bar{g}_{\#}\right)_{b}$. 
But now $C\left(\bar{f}_{\#}, \bar{g}_{\#}\right)=0$ so this orbit is a class from $\nabla_{K}\left(f_{b}, g_{b}\right)$ and since $K=0, A \cap E_{b} \in \nabla\left(f_{b}, g_{b}\right)$ is a Nielsen class of $\left(f_{b}, g_{b}\right)$. Since $A \cap E_{b}$ contains a self-reducing point of $\left(f_{b}, g_{b}\right)$, it is defective and $\mid$ ind $\mid\left(f_{b}, g_{b}: A \cap E_{b}\right) \leq 1$. But as we have noticed, ind $(\bar{f}, \bar{g}: p A)= \pm 1$, hence the assumption (a) of (3.13) is satisfied and SIPF follows.

5. An application. Now we apply the formulae of Section 4 to determine the Nielsen numbers of maps of some $K(\pi, 1)$ spaces. The main result of this section is Corollary (5.5).

(5.1) Definition. Let $\phi: M \rightarrow M$ be a diffeomorphism of a smooth $n$-manifold. We define $S_{\phi}=M \times I / \sim$ where $\sim$ identifies the points $(m, 0)$ and $(\phi(m), 1)$. Then $S_{\phi}$ is a locally trivial fibre bundle over the circle $S^{1}=$ $[0,1] /\{0,1\}$.

Let $\phi^{\prime}: M^{\prime} \rightarrow M^{\prime}$ be another diffeomorphism and $\operatorname{dim} M=\operatorname{dim} M^{\prime}$. We consider a commutative square

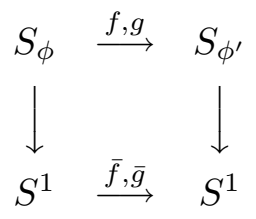

Let ind $(\bar{f}, \bar{g})=k$. If $k=0$ then $N(\bar{f}, \bar{g})=0$. Now let $k \neq 0$. To simplify the notation we will assume that $k>0$ (otherwise we may consider $(g, f)$ ). Then $N(\bar{f}, \bar{g})=k$ and we may assume that $(f, g)$ has exactly $k$ coincidence points, $\Phi(f, g)=\left\{b_{0}, \ldots, b_{k-1}\right\}$. We also fix a path $\bar{u}_{i}$ from $b_{0}$ to $b_{i}$ such that $\operatorname{deg}\left(\overline{g u}_{i}-\bar{f} \bar{u}_{i}\right)=i(i=1, \ldots, k-1)$. Then the homotopy commutative diagram

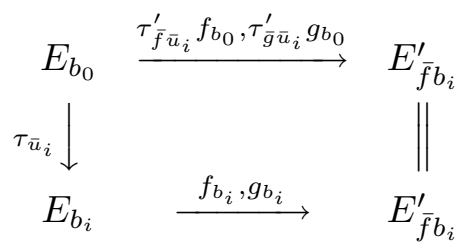

and (4.4) imply

$$
\begin{aligned}
N\left(f_{b_{i}}, g_{b_{i}}\right) & =N\left(\tau_{\bar{f} \bar{u}_{i}}^{\prime} f_{b_{0}}, \tau_{\bar{g} \bar{u}_{i}}^{\prime} g_{b_{0}}\right)=N\left(f_{b_{0}},\left(\tau_{\bar{f} \bar{u}_{i}}^{\prime}\right)^{-1} \tau_{\bar{g} \bar{u}_{i}}^{\prime} g_{b_{0}}\right) \\
& =N\left(f_{b_{0}}, \tau_{\bar{g} \bar{u}_{i}-\bar{f} \bar{u}_{i}}^{\prime} g_{b_{0}}\right)=N\left(f_{b_{0}}, \phi^{\prime i} g_{b_{0}}\right) .
\end{aligned}
$$

Now (4.4) implies

$$
N(f, g)=\sum_{i=0}^{k-1} N\left(f_{b_{i}}, g_{b_{i}}\right)=\sum_{i=0}^{k-1} N\left(f_{b_{i}}, \phi^{\prime i} g_{b_{0}}\right) .
$$


The following three lemmas give us criteria to decide whether a given map is homotopic to a fibre map.

(5.2) LEMmA. Let $(E, p, B),\left(E^{\prime}, p^{\prime}, B^{\prime}\right)$ be locally trivial fibre bundles in which all spaces are $K(\pi, 1)$ 's. Then a map $f: E \rightarrow E^{\prime}$ is homotopic to a fibre map iff the composition $p_{\#}^{\prime} f_{\#} i_{\#}: \pi_{1} E_{b} \rightarrow \pi_{1} E \rightarrow \pi_{1} E^{\prime} \rightarrow \pi_{1} B^{\prime}$ is zero.

Proof. The necessity is obvious. Now suppose that $p_{\#}^{\prime} f_{\#} i_{\#}=0$. Consider the diagram with exact rows

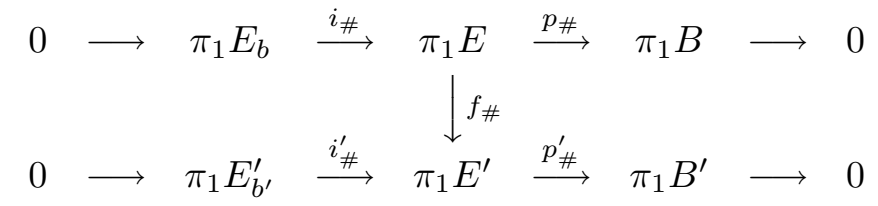

Let $\langle a\rangle \in \pi_{1} E_{b}$. Since $p_{\#}^{\prime} f_{\#} i_{\#}\langle a\rangle=0$, we have $f_{\#} i_{\#}\langle a\rangle \in \operatorname{ker} p_{\#}^{\prime}=\operatorname{im} i_{\#}^{\prime}$ and there exists a unique $\left\langle a^{\prime}\right\rangle \in \pi_{1} E_{b^{\prime}}^{\prime}$ such that $i_{\#}^{\prime}\left\langle a^{\prime}\right\rangle=f_{\#} i_{\#}\langle a\rangle$. We define a homomorphism $h_{0}: \pi_{1} E_{b} \rightarrow \pi_{1} E_{b^{\prime}}^{\prime}$ setting $h_{0}\langle a\rangle=\left\langle a^{\prime}\right\rangle$. Now $h_{0}$ and $f_{\#}$ determine a homomorphism $\bar{h}$ such that the diagram

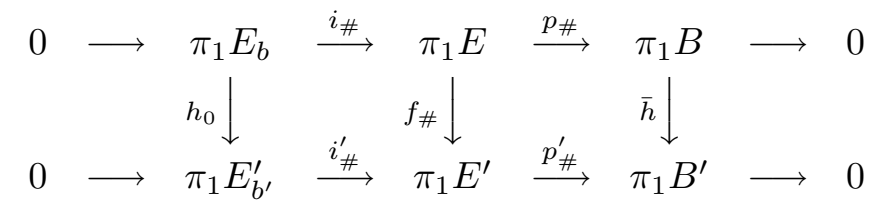

is commutative. Since $B$ and $B^{\prime}$ are $K(\pi, 1)$ spaces therefore $\bar{h}=\bar{f}_{\#}$ for some $\bar{f}: B \rightarrow B^{\prime}$. Then $\bar{f}_{\#} p_{\#}=p_{\#}^{\prime} f_{\#}$ implies that $p^{\prime} f$ is homotopic to $\bar{f} p$. Let $\bar{H}: E \times I \rightarrow B^{\prime}$ denote this homotopy and consider the diagram

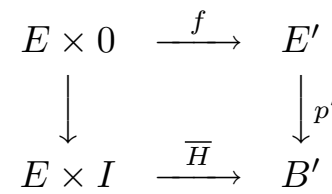

Then $\bar{H}$ can be lifted to some $H: E \times I \rightarrow E^{\prime}$. We put $f_{1}=H(\cdot, 1): E \rightarrow E^{\prime}$ and get the desired fibre map homotopic to $H(\cdot, 0)=f$.

(5.3) Lemma. Let $(E, p, B),\left(E^{\prime}, p^{\prime}, B^{\prime}\right)$ be fibre bundles with all spaces $K(\pi, 1)$ 's. Let $\bar{u}$ be a loop based at $b \in B$. Assume that whenever the diagram

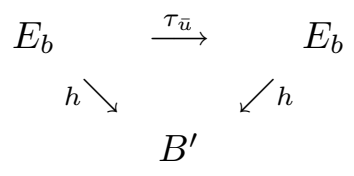


is homotopy commutative then $h$ is homotopic to a constant map. Then any $f: E \rightarrow E^{\prime}$ is homotopic to a fibre map.

Proof. The diagram

$$
\begin{array}{rll}
E_{b} & \stackrel{\tau_{\bar{u}}}{\longrightarrow} & E_{b} \\
p^{\prime} f_{\mid E_{b}} \searrow & B^{\prime} & \\
& B^{\prime} f_{\mid E_{b}}
\end{array}
$$

is homotopy commutative by $H(x, t)=p^{\prime} f \tau_{\bar{u}_{0}^{t}}(x)$ (here $\bar{u}_{0}^{t}$ is the path given by $\left.\bar{u}_{0}^{t}(s)=\bar{u}(t s)\right)$. Now by our assumption $p^{\prime} f_{\mid E_{b}}$ is homotopic to a constant map and (5.2) implies our lemma.

(5.4) LEMMA. Let $\phi, \phi^{\prime}$ be diffeomorphisms of a $k$-dimensional torus and let $C$ be a $k \times k$ matrix representing $\phi_{\#}: \pi_{1} T \rightarrow \pi_{1} T$. Let $\operatorname{det}(I-C) \neq 0$ (i.e. 1 is not an eigenvalue of $C$ ). Then any continuous map $f: S_{\phi} \rightarrow S_{\phi^{\prime}}$ is homotopic to a fibre map.

Proof. We consider the diagram from (5.3) for $\bar{u}$ a loop of degree one:

$$
\begin{gathered}
T \stackrel{\tau_{\bar{u}}}{\longrightarrow} T \\
p^{\prime} f_{\mid T} \searrow \\
S^{1}
\end{gathered}
$$

But $\left(\tau_{\bar{u}}\right)_{\#}=\phi_{\#}$ is represented by $C$ and let $h$ be a $1 \times k$ matrix representing $\left(p^{\prime} f_{\mid T}\right)_{\#}: \pi_{1} T \rightarrow \pi_{1} S^{1}$. Now the above diagram implies $h C=h$, so that $h(C-I)=0$ and the assumption $\operatorname{det}(I-C) \neq 0$ implies $h=0$. Now the lemma follows from (5.3).

Finally, we sum up the results of this section in

(5.5) Corollary. Let $\phi, \phi^{\prime}$ be diffeomorphisms of the $k$-dimensional torus. Let $k \times k$ matrices $C, D$ represent $\phi_{\#}, \phi_{\#}^{\prime}: \pi_{1} T \rightarrow \pi_{1} T$ and suppose $\operatorname{det}(I-C) \neq 0$. Then any pair of continuous maps $f, g: S_{\phi} \rightarrow S_{\phi^{\prime}}$ is homotopic to a fibre pair

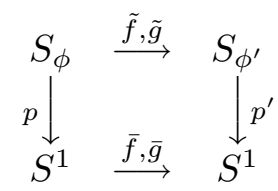

If ind $(\bar{f}, \bar{g})=0$ then $(\bar{f}, \bar{g})$ is homotopic to a coincidence free pair, hence so is $(f, g)$ and $N(f, g)=0$. If $\operatorname{ind}(f, g)=k \neq 0$ then fix a coincidence point $b \in S^{1}$ and denote by $A$ and $B$ the matrices representing $\widetilde{f}_{b \#}$ and $\widetilde{g}_{b \#}$. Then

$$
N(f, g)=\sum_{i=0}^{|k|-1} N\left(f_{b}, \phi^{\prime i} g_{b}\right)=\sum_{i=0}^{|k|-1}\left|\operatorname{det}\left(A-D^{i} B\right)\right| .
$$


(5.6) Re mark. (i) If, in (5.5), we have $S_{\phi}=S_{\phi^{\prime}}, f=$ id then we obtain a new formula for the fixed point Nielsen number of a map $g: S_{\phi} \rightarrow S_{\phi}$ : if $\operatorname{deg} \bar{g}=k \neq 1$ then $\operatorname{ind}(\bar{g})=k-1$ and

$$
N(g)=\sum_{i=0}^{|k-1|-1} N\left(\phi^{\prime i} g_{b}\right)=\sum_{i=0}^{|k-1|-1}\left|\operatorname{det}\left(E-D^{i} B\right)\right|
$$

whereas if $\operatorname{deg} \bar{g}=1$ then $N(g)=0$.

(ii) The assumptions of (5.5) are satisfied for the oriented flat 3-manifolds $\mathcal{G}_{2}, \ldots, \mathcal{G}_{5}$ of $[\mathrm{W}, \mathrm{Thm} .(3.5 .5)]$. The projection on the first coordinate makes these manifolds fiberings over $S^{1}$. Each of them becomes an $S_{\phi^{\prime}}$ for $\phi^{\prime}$ a self-map of the 2-torus such that $\phi_{\#}^{\prime}$ is represented by

$$
\left[\begin{array}{ll}
a_{22} & a_{23} \\
a_{32} & a_{33}
\end{array}\right]
$$

where $A=\left[a_{i j}\right]_{i, j=1,2,3}$ denotes the corresponding orthogonal map from [W, (3.5.5)].

(iii) Corollary (5.5) generalizes Section 3 in [DJ]: the Klein bottle may be represented as $S_{\phi}$ for $\phi: S^{1} \rightarrow S^{1}$ given by $\phi(x, y)=(x,-y)$.

\section{References}

[DJ] R. Dobreńko and J. Jezierski, The coincidence Nielsen number on non-orientable manifolds, Rocky Mountain J. Math., to appear.

[H] M. Hirsch, Differential Topology, Springer, New York 1976.

[Je] J. Jezierski, The Nielsen number product formula for coincidences, Fund. Math. 134 (1989), 183-212.

[J] B. J. Jiang, Lectures on the Nielsen Fixed Point Theory, Contemp. Math. 14, Amer. Math. Soc., Providence, R.I., 1983.

[V] J. Vick, Homology Theory, Academic Press, New York 1976.

[W] J. A. Wolf, Spaces of Constant Curvature, Univ. of California, Berkeley 1972.

[Y] C. Y. You, Fixed points of a fibre map, Pacific J. Math. 100 (1982), 217-241.

DEPARTMENT OF MATHEMATICS

UNIVERSITY OF AGRICULTURE

NOWOURSYNOWSKA 166

02-766 WARSZAWA, POLAND 